This Accepted Manuscript is available for reuse under a CC BY-NC-ND 3.0 licence after the 12 month embargo period provided that all the terms of the licence are adhered to. This is a peer-reviewed, un-copyedited version of an article published in Measurement Science and Technology. IOP Publishing Ltd is not responsible for any errors or omissions in this version of the manuscript or any version derived from it. The Version of Record is available online at https://doi.org/10.1088/1361-6501/aad1e1. 


\title{
A 3-D measuring endoscope for hand-guided operation
}

\author{
Steffen Matthias, Markus Kästner, Eduard Reithmeier \\ Institute of Measurement and Automatic Control, Leibniz Universität Hannover, \\ Nienburger Str. 17, 30167 Hannover, Germany \\ E-mail: steffen.matthias@imr.uni-hannover.de \\ January 2018
}

\begin{abstract}
Optical measuring devices are widely used in industrial production and maintenance processes. With increasing integration, the accessibility of functional elements inside machines is limited for measuring devices. In order to provide a new measurement tool for these applications, the design of an endoscopic 3-D sensor based on the structured light principle is presented. Application specific sensor heads are introduced, with diameters down to $8 \mathrm{~mm}$ attached to endoscopes with a length of $1 \mathrm{~m}$. The sensor features a flexible pattern generator and a camera, allowing for dynamic reconfiguration of structured illumination patterns. Based on a brief comparison of existing light coding approaches, two different measuring modes for the sensor are described. The first measuring mode is using conventional phase-shift sequences in order to achieve highly accurate measurements under controlled conditions. The second measuring mode uses a new interleaved encoding approach for phase-shift patterns, which enables robust measurements even in the presence of relative motion between object and sensor. An example for this type of motion is the movement of the endoscope tip by hand. Using the proposed sequence design, the 3-D acquisition rate is identical to the capture rate of the camera, which amounts to a maximum of $180 \mathrm{~Hz}$ in the current hardware configuration of the measuring endoscope. In combination with a registration and merging algorithm, consecutive measurements are automatically combined, enabling the measurements of large surfaces. Moreover, the approach is capable of estimating the relative location and orientation of the endoscope tip using odometry based on depth data. The performance of the sensor in both measuring modes is evaluated on the basis of measurements of reference objects. Furthermore, the results of a hand-held measurement of a reference standard are shown.
\end{abstract}

Keywords: 3-D endoscopy, structured light, motion, odometry

\section{Introduction}

Dimensional measurements are a crucial part in the quality control of modern industrial production and maintenance processes [1]. Owing to their non-destructive nature and high measurement speed, optical methods have been established next to tactile 
surface measuring methods [2]. A variety of techniques are used in industrial applications to capture areal geometry and surface topology data in different scales. For the measurement of surface roughness parameters, typically microscopes using interferometric or confocal methods are used. In order to measure the geometry of larger surface areas at a reduced resolution, for example in industrial manufacturing processes, structured light sensors are widely used [3, 4]. The measurement duration of structured light sensors based on the triangulation principle is typically in the order of seconds or below, enabling the use as inline measuring devices. Furthermore, both lateral and depth resolution is higher at shorter ranges compared to sensors based on the time-of-flight (ToF) principle [5].

However, available optical sensors are not applicable for machines where crucial geometries are not accessible on the outside. An example for these machines are aircraft engines. In order to avoid the cost and time of disassembly, the interior of the engines is inspected using industrial endoscopes, also called borescopes. On-wing maintenance processes for air-plane engines are mostly relying on visual inspection by schooled operators [6]. Owing to safety and economic reasons, a move to reliable assessments based on 3-D measurements is desired. Similar problems and difficulties regarding the inline application of sensors can also be found in industrial manufacturing processes. Weckenmann et al. state the miniaturization of sensors as one of the key challenges in industrial metrology [7].

For this reason, multiple approaches for the combination of optical 3-D measuring methods and endoscopy techniques have been investigated in literature. For medical applications, an overview over 3-D measuring endoscopes in literature is given by Geng and Xie [8] including a discussion of different designs for structured light sensors.

Noonan et al. present a stereo-vision based approach for the reconstruction of depth positions and the estimation of camera motion [9]. Two independent fibreoptical endoscopes are used to capture stereo images at the tip. Due to the use of photogrammetry, the feasibility of the approach is dependent on the texture of the measuring object. According to the authors, reconstruction errors for a biological tissue are in the range of a few millimetres.

Penne et al. developed a prototype of a ToF based measuring endoscope [10]. An industrial ToF camera is coupled to a rigid endoscope to acquire depth images with a capture rate of $25 \mathrm{~Hz}$. Depth images feature a lateral resolution of $64 \times 48$ pixel. Length measurement errors of the sides of a reference cube amount to less than $1 \mathrm{~cm}$.

Hasegawa et al. [11] demonstrate a flexible measuring endoscope consisting of a fibre-coupled projector and chip-on-tip camera. The diameter of the endoscope tip is $16 \mathrm{~mm}$ and features a triangulation base length of $8.5 \mathrm{~mm}$. Measurements of planar reference targets exhibit a standard deviation of approximately $310 \mu \mathrm{m}$.

Pulwer et al. [12] describe the design of an endoscope with structured illumination. Patterns are generated at the tip of the endoscope and captured by a chip-on-tip camera. The visual operation of the endoscope in an industrial application is demonstrated. However, no measurement results are provided. 
The Storz Multipoint Videoscope is a commercially available endoscope by KARL STORZ GmbH \& Co. KG (Tuttlingen, Germany), which uses the laser point triangulation principle to acquire $493-\mathrm{D}$ measurement points on a technical surface [13]. No areal triangulation is possible with the type of structured illumination used.

General Electric (Boston, USA) developed a phase measuring endoscope for industrial applications (XLG3 Videoscope) [14]. The system is capable of capturing areal 3-D measurements of surfaces, while featuring compact sensor heads. According to Korczewski, the system is limited by the measurement duration of approximately $2 \mathrm{~s}$, which impairs the applicability for manual inspection [15].

In general, next to accuracy, a limitation of most of the previously described approaches is their inability to obtain reliable measurement results under the influence of motions. However, industrial endoscopes are usually operated by hand, hence motion cannot be avoided.

In order to improve on existing approaches regarding measurement uncertainty and measurement speed, in this contribution the design of a new fibre-endoscopic structured light sensor is presented. Highly capable camera and pattern projection components are coupled to imaging fibre bundles. This design enables both very compact sensor heads and flexible projection patterns. The patterns can be dynamically reconfigured in order to balance between measurement accuracy and measurement duration. In combination with the proposed new pattern coding scheme, dense 3-D geometry data can be measured at high rates. In both measuring modes the measuring results can be provided in the form of a depth map or a 3-D point cloud. Additionally, an intensity image corresponding to each depth map is provided by the measuring algorithm.

The first section of this publication introduces the hardware components of the fibre-endoscopic sensor. Subsequently, the second section gives a brief overview on commonly used structured light patterns. After a description of the conventional measurement mode based on phase-shift patterns, a new periodic interleaved projection sequence for fast continuous measurement is proposed. Section 4 shows measurement results obtained with the sensor in both the conventional and in the continuous measurement mode. A discussion of the results is given in Section 5 , followed by a summary and an outlook in Section 6.

\section{Hardware components}

The new 3-D measuring endoscope consists of a projection and camera unit and fibreoptic endoscopes to transport pattern images to compact sensor heads. Image fibres of type FIGH are supplied by Fujikura Ltd. (Tokyo, Japan) and feature 50000 or 100000 cores over a length of $1000 \mathrm{~mm}$. The fibres with higher core count exhibit an image diameter of $1.4 \mathrm{~mm}$, while the fibres with lower core count exhibit a diameter of $1.0 \mathrm{~mm}$. For both types of fibre bundles, the average core-to-core spacing is approximately $4 \mu \mathrm{m}$ and the numerical aperture is 0.4 [16]. A schematic view of the sensor is shown in Fig. 1, with the sensor head on the left and the pattern detector and generator components 


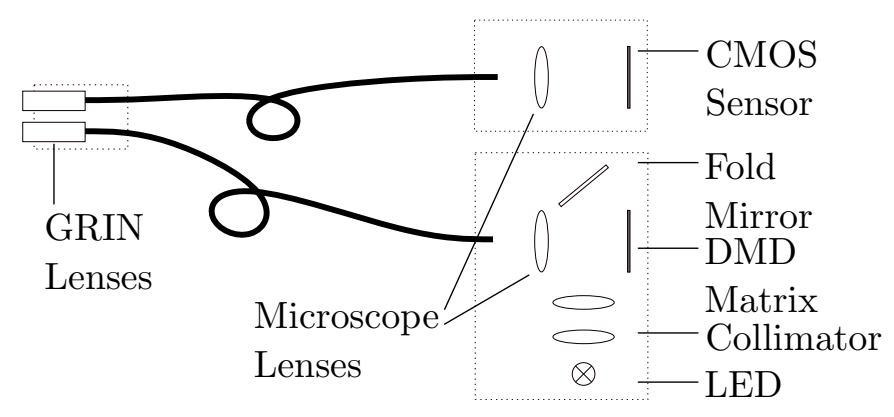

Figure 1. Schematic drawing of sensor components (not to scale).

\begin{tabular}{|l|c|c|}
\hline Working distance & $10 \mathrm{~mm}$ & $20 \mathrm{~mm}$ \\
\hline Approx. field diameter & $6 \mathrm{~mm}$ & $12 \mathrm{~mm}$ \\
\hline Approx. depth-of-field & $2 \mathrm{~mm}$ & $6 \mathrm{~mm}$ \\
\hline Approx. lateral resolution & $20 \mu \mathrm{m}$ & $40 \mu \mathrm{m}$ \\
\hline
\end{tabular}

Table 1. Measuring volumes of typical lens configurations for fibres with $1.4 \mathrm{~mm}$ image diameter.

on the right. In the current configuration, the sensor uses an industrial camera of type GS3-U3-23S6M-C by FLIR Systems (Wilsonville, USA), which is capable of a maximum full-frame acquisition rate of $162 \mathrm{~Hz}$. The projector is using a DLP4100 development kit supplied by Vialux (Chemnitz, Germany) in combination with a self-developed LED illumination and achieves a maximum gray-scale pattern rate of $252 \mathrm{~Hz}$. For both camera and projector, fibre coupling is performed using microscope objectives.

In the sensor head designs, typically gradient-index (GRIN) rod lenses by GRINtech (Jena, Germany) with a diameter of $2 \mathrm{~mm}$ or less are used. Depending on the application, different working distances are possible. In most cases, either lenses with a working distance of $10 \mathrm{~mm}$ or $20 \mathrm{~mm}$ are employed. By combining the lenses with mirror prisms, optionally sideways facing measurements can be achieved. Typical configurations are listed in Tab. 1. It is possible to obtain measurements outside of the specified depth ranges at a reduced resolution resulting from image blur.

Owing to the compact dimensions of both imaging fibres and lenses, compact designs can be achieved for the sensor heads. Fig. 2 shows a compact sensor head with a diameter of $8 \mathrm{~mm}$, featuring a triangulation base length of $3.5 \mathrm{~mm}$. The sensor head shown in Fig. 3 shows a slightly larger sensor head with a height of $15 \mathrm{~mm}$ and a triangulation base length of $6 \mathrm{~mm}$. The size is a result of the larger metal sleeves added to the end segments of the fibres by the manufacturer. Furthermore, currently screws are used to enable a flexible change of fibre arrangement and optics. By using different manufacturing processes, i.e. directly gluing the fibres to the sensor heads, significantly smaller sensor head designs could be achieved.

Both sensor heads feature a similar measuring performance, as the fibre core spacing and diameter is identical. However, the fibres used for the smaller sensor head has a $29 \%$ smaller field diameter due to the reduced fibre image diameter of $1 \mathrm{~mm}$. 


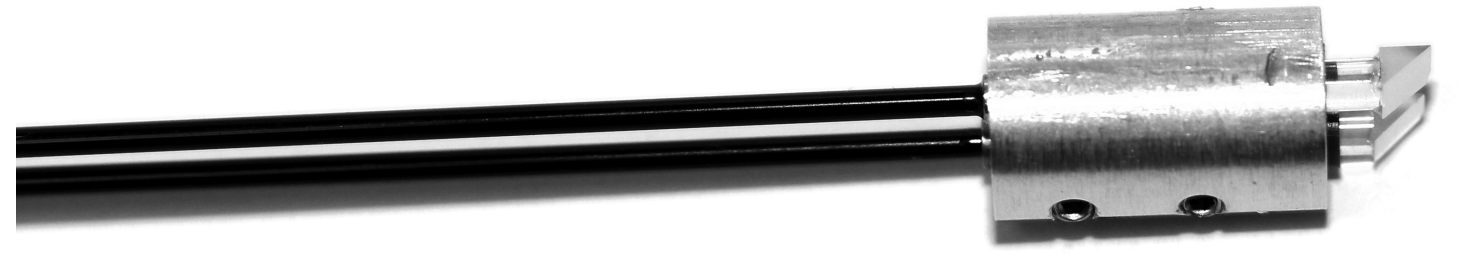

Figure 2. Compact sensor head with mirror prisms (diameter: $8 \mathrm{~mm}$ ).

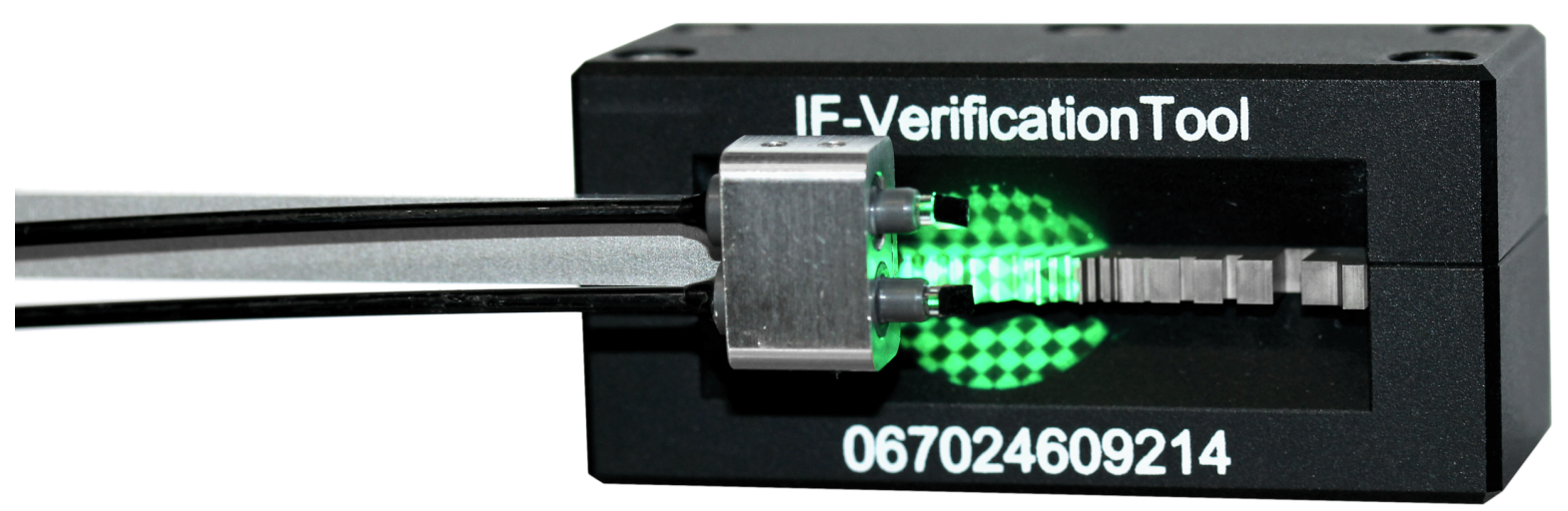

Figure 3. Larger sensor head (dimensions: $8.5 \times 15 \times 13 \mathrm{~mm}^{3}$ ) projecting patterns onto a micro-contour standard.

Fig. 4 shows a camera image taken by the endoscope. The image has been processed using an adaptive histogram equalization algorithm to reduce the high dynamic range of the input image for visualization. Visible in the image are slight pixelation artefacts arising from the discrete structure of the fibre bundles. Furthermore, the field curvature of the gradient-index lenses is evident in the form of a slight blur to the edges of the window. Owing to the dense packing of cores in the fibre bundles, inter-core coupling of light occurs, resulting in a loss of image contrast. The effect is visible in the camera images especially in proximity to the geometry features. In order to avoid influences on measurements, a combination of two compensation techniques was developed. Accurate measurements of the transfer properties of imaging fibres enable the reconstruction of camera images using deconvolution algorithms [17]. Furthermore, a high dynamic range imaging approach with adaptive adjustment of the projection patterns is applied to further reduce the influences of cross-talk [18]. 


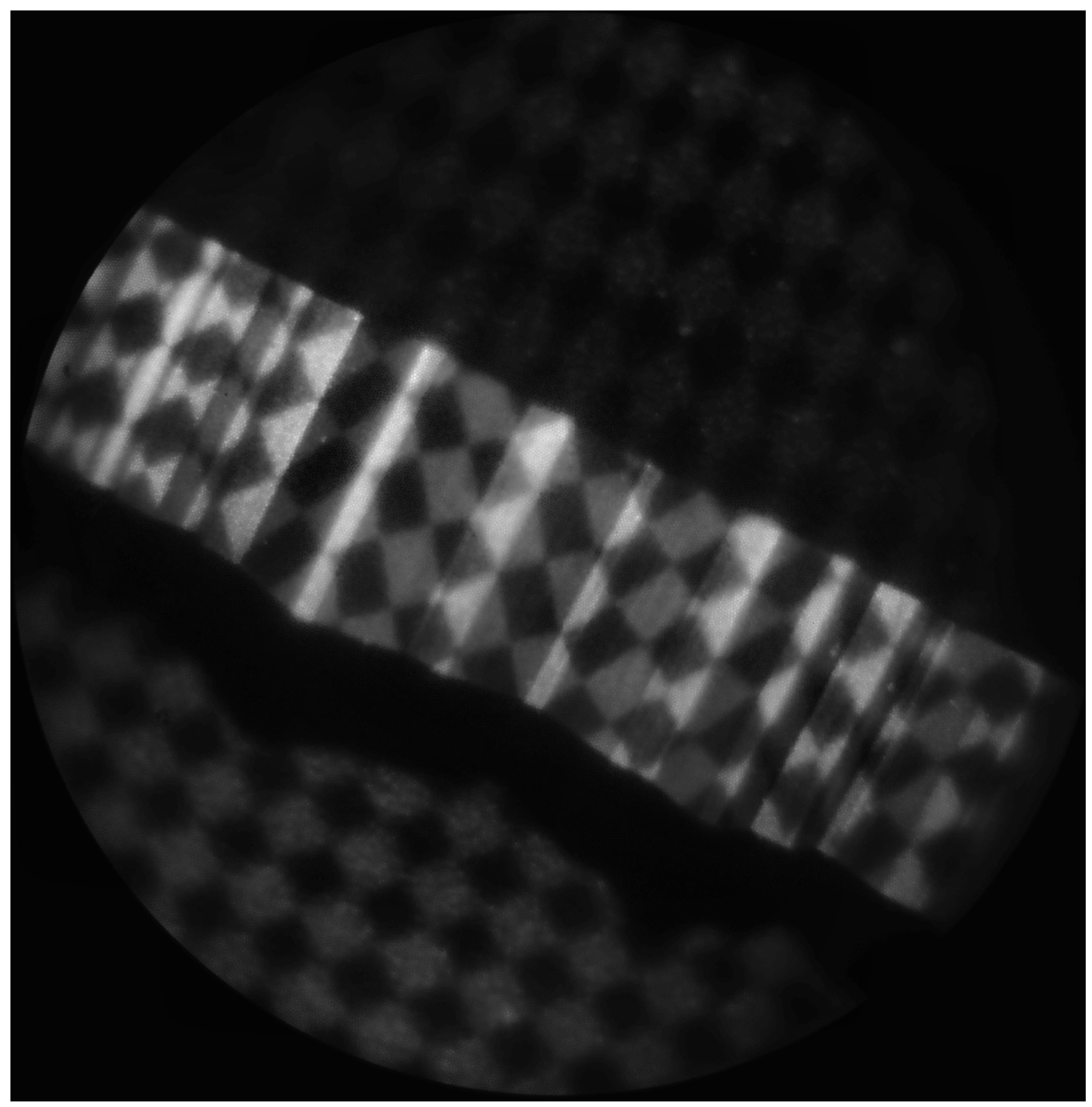

Figure 4. Endoscope view of micro-geometries with chequerboard pattern projection.

\section{Measuring modes}

Two measuring modes are implemented for the structured light sensor with different performance characteristics. The goal of both modes is the acquisition of 3-D geometry information, which is provided in the form of a 3-D point cloud or alternatively a depth map. During the measuring process, a sequence of structured light patterns is synchronously projected onto a specimen and captured by the camera. The type of patterns used is having a critical influence on the measurement result, i.e. a depth map or 3-D point cloud, because they are used to establish correspondences between camera and projector of the sensor for subsequent triangulation. The first measuring mode uses conventional phase-shift sequence combined with a heterodyne phase unwrapping 
approach. For the fast measurement of consecutive 3-D datasets, a new sequence design based on phase-shift patterns is proposed.

Subsequently, the acquired pattern images are analyzed by algorithms for absolute phase estimation, which will later be described in more detail. On the basis of the recovered phase-maps, correspondences between camera and projector of the sensor can be established. Subsequently, triangulation of data points is performed based on the camera-projector correspondences acquired. The implemented algorithms use the pinhole model [19] in combination with the Brown-Conrady lens distortion model [20] for both camera and projector.

In order to motivate the development of the two different measuring modes, this section gives a brief overview on structured light coding approaches. The chosen coding approach influences the accuracy of the obtained camera-projector correspondences required for triangulation of measurement points. Furthermore, it defines the acquisition time of 3-D measurements and the robustness towards environmental influences, such as surface texture or external light sources. A detailed overview on pattern coding strategies is given by Salvi et. al [21]. In general, the projection of longer pattern sequences usually results in lower noise compared to shorter sequences or single pattern approaches. However, when relative motion between specimen and sensor is present, multi-pattern sequences suffer from motion artifacts due to the longer acquisition time required.

Section 3.1 discusses the pattern sequences employed for the slower conventional measuring mode of the new sensor. The discussed type of patterns is generally very robust to influences of the environment, as long as no motion between sensor and measuring object is present. Section 3.2 describes a new approach for a periodic phase-shift sequence, which allows for the fast consecutive acquisition of multiple 3D measurements. Compared to sinusoidal single-pattern approaches, as described for example by Takeda et al. [22], the sequence is more robust towards environmental influences. Pseudo-random patterns, as introduced for example by Zhang et al [23], exhibit a lower lateral resolution due to the neighborhood operation for the local correlation analysis.

Multi-frequency phase-shift sequences consist of multiple cosine-modulated patterns. As these patterns feature multiple cosine-periods to increase robustness, the phase recovered from them is by itself not un-ambiguous. This intermediate phase-map is a relative phase map, which is wrapped to a range of $2 \pi$. In a second step, two or more relative phase maps obtained from patterns with different frequencies are combined to obtain an absolute phase map with unambiguous values. In both described measuring modes, phase unwrapping is performed using a heterodyne multi-frequency approach. An overview over different multi-frequency unwrapping techniques is given by Zuo et al. [24]. Multi-frequency unwrapping algorithms typically requires less patterns than earlier techniques based on Gray-codes as discussed by Sansoni et. al [25]. 


\subsection{Conventional phase-shift sequences}

For measurements of non-moving objects conventional phase-shift sequences in combination with temporal phase-unwrapping are used. Owing to the optical properties of the imaging fibre bundles, a temporal phase-unwrapping approach based on heterodyne evaluation has shown to increase robustness for measurements using the endoscope [17]. The heterodyne-based approach avoids the projection of low-frequency patterns and therefore reduces cross-talk in the fibre bundles compared to other temporal unwrapping approaches.

In order to obtain accurate relative phase-maps prior to phase-unwrapping, at least three phase-shift patterns are projected for the highest pattern frequency, followed by an evaluation using the symmetrical n-step algorithm [26].

Parametrization options in the conventional mode are the number of frequencies used in the unwrapping process as well as the number of phase-shift patterns. Both parameters influence the length of the projection sequence. For the measuring endoscope, the use of three frequencies for phase unwrapping has shown robust results. In general, an increase in the number of phase-shifts for the highest pattern frequency results in an increase in the signal-to-noise-ratio for the subsequent phase evaluation. The lower frequency patterns used for phase-unwrapping use a reduced number of two phase-shifts according to the approach described by Peng and Gupta [27]. In most measurement situations, a sequence length of 8 patterns is sufficient for acquiring reliable measurements.

In order to allow the measurement of highly reflective objects, pattern sequences are captured at multiple exposure times. This allows for the combination to high dynamic range pattern images [28] and therefore robust phase evaluation.

\subsection{Interleaved three-pattern phase-shift sequence}

Next to the single-shot measuring mode based on conventional phase-shift sequences, a continuous measuring mode has been implemented for the endoscopic sensor. This mode is capable of capturing full-field 3D measurements at a rate identical to the camera acquisition rate using a special periodic projection sequence.

The general approach for the new sequence is the reduction of a conventional phaseshift sequence to three total patterns. In order to motivate the design of the sequence, initially general properties of phase-shift pattern sequences are discussed. These type of sequences are commonly used for structured light 3-D sensors [27] and include the sequences described in Section 3.1. The description is based on the previous introduction of the sequence design by the authors in [29].

As described in Section 3, phase recovery is performed in a two step process. Initially, the process for recovering the locally wrapped, i.e. relative, phase is described. Afterwards, the sequence design for acquiring a absolute phase maps is derived.

The intensity at a given detector pixel $g_{n}(x, y)$ of a phase shift sequence of length 
$N$ can be expressed by

$$
g_{n}(x, y)=a(x, y)+b(x, y) \cos \left(\phi(x, y)+\frac{n 2 \pi}{N}\right)
$$

where $a$ is the background illumination and constant pattern component and $b$ is the amplitude modulation component [30]. Both modulations are resulting from reflectivity and texture of a surface and vary over the camera image. The local phase of the pattern is described by $\phi(x, y)$, while the patterns with index $n=0 \ldots N-1$ are shifted equally over $2 \pi$.

For diffuse reflective specimen, $a$ and $b$ exhibit low bandwidth in frequency domain, allowing for separation of the components and the recovery of the relative local phase by assuming low variation in $b$ compared to the local modulation of the phase $\Delta \phi$, as described by Takeda et. al [22]. However, if the specimen features varying texture and reflectivity, which is the case for almost all technical surfaces, spectrum components of $a$ and $b$ overlap as the multiplication results in a convolution in frequency domain. As a result, a higher pattern count needs to be chosen for the projection sequence, with a pattern count of $N \geq 3$. The approach for the conventional measuring mode, described in Section 3.1, follows this robust measurement approach. For fewer patterns than 3, not all components can be separated if the previously discussed overlap of pattern components in Fourier domain is present. In this case, phase recovery without large distortions is not possible. A discussion of phase-shift patterns in Fourier domain is provided by the authors in [29]. In general, patterns are affected by the following influences:

- vignetting $(a, b)$

- defocus $(a, b)$

- ambient light $(a)$

- surface reflectivity and texture $(a, b)$

Under practical conditions, surface reflectivity and texture is the dominating influence on both $a$ and $b$. Ambient light is typically not directed onto the surface and is for most sensors significantly lower in intensity than the light emitted by the projector. Considering the normalized intensity of a pixel of the projected pattern with index $n$ in the pattern sequence with length $N$ is described by

$$
i_{(p, n)}=\frac{1}{2}+\frac{1}{2} \cos \left(\phi+\frac{n 2 \pi}{N}\right),
$$

where $\phi$ describes the phase corresponding to the pattern point. Assuming the point on the projector pattern is reflected from the object's surface to a point on the camera, the intensity for the corresponding camera pixel can be described by

$$
i_{(c, n)}=i_{0}+f_{r} i_{(p, n)},
$$

where $i_{0}$ is the ambient illumination and $f_{r}$ is the ratio of light which is reflected from projector to camera for the given point. The ratio is depending on the bidirectional reflectance distribution function, and typically varies for each imaged surface point due 
to different surface, illumination and observation angles as well as texture. Using this simple model, it can be derived that $a=i_{0}+\frac{1}{2} f_{r}$, while $b=\frac{1}{2} f_{r}$. Both parameters are influenced by the reflection ratio $f_{r}$, while $a$ is additionally influenced by the ambient illumination $i_{0}$. As the pattern projector of the structured light sensor is brighter than typical ambient illumination, $i_{0}$ is much smaller than $\frac{1}{2} f_{r}$. Consequently, $a$ provides a good estimate for parameter $b$, as both a dominated by $f_{r}$. Vignetting, which can occur with certain camera and projector optics, has similar effects on $a$ and $b$, while defocus has more effect on $b$ due the modulated illumination and therefore higher frequency components. Defocus is mathematically modeled by convolution of the image with a point spread function and results in low-pass filtering of the acquired images. As a result, surface reflectivity affects the local phase recovery process and leads to measurement deviations [17].

As previously noted in Section 3.1, local phase recovery is typically performed by obtaining a phase-shift sequence of at least $N=3$ patterns. However, in order to reduce measurement times, shorter sequences are preferred. The applicability of sequences with $N=1$, i.e. the so-called Fourier transform method [22], is limited if the surface reflectivity is varying.

For the case of $N=2$, the robustness of local phase recovery can be significantly improved compared to the case of $N=1$ by following the subsequently described steps. Initially, the constant pattern components $a$ can be estimated by calculating the sum of two pattern images $g_{0}$ and $g_{1}$ with a relative phase offset of $\pi$ according to $a=g_{0}+g_{1}$.

Subsequently, the phase shift patterns are divided in spatial domain by $a$, which is equal to the deconvolution of reflectivity and texture in frequency domain. Thus it results in a bandwidth reduction of the two modulated, i.e. cosine, pattern components present in the signal. As ideally no remaining overlap in the frequency domain is present after this step, one side of the spectrum can be suppressed by employing a mask in the frequency domain. After applying the inverse Fourier transform to the bandpass filtered spectrum, a complex signal is obtained, from which the local phase of the sequence with $N=2$ can be obtained. As long as influences of defocus and ambient light are kept small, the phase can accurately be recovered. This last filtering step is similar to the approach described by Takeda and Mutoh for single sinusoidal patterns [22]. Owing to the added second pattern, the newly described approach allows to estimate and compensate the influence of surface texture and therefore significantly increases robustness. Furthermore, the approach is capable of achieving a robustness similar to the three-step algorithm, as described by K. Creath [30], under typical measuring conditions.

The previous properties lead to the design of the proposed three-pattern absolute phase algorithm. Two patterns of same frequency with a relative phase-shift of $\pi$ are required to estimate the per-pixel surface reflectivity and a relative phase-map. A third pattern of a second frequency is added to allow for robust unwrapping of the absolute phase without requiring constraints on the object's surface or sensor design. For this second frequency, phase retrieval is performed similarly, by using the previously 
obtained estimation for surface reflectivity and texture. Unwrapping of the two relative phase-maps to an absolute phase-map is based on the heterodyne approach described by Towers et. al [31].

Following the described approach, full-field absolute phase retrieval is possible from three patterns with the following properties: Two patterns feature a phase offset of $\pi$ and identical frequency, while the third pattern with arbitrary phase offset features a frequency differing from the other two patterns to allow phase unwrapping. A periodic sequence can be derived from these constraints, in which any three neighboring patterns fulfill the requirements for the outlined three-pattern algorithm. Six patterns of the pattern sequence with an exemplary frequency are shown in Fig. 5. As illustrated, patterns of two frequencies and phase offsets are interleaved to be projected alternately. The sequence has a period length of four patterns, which are highlighted by the dashed box. In order to achieve the desired measurement count, the four patterns can be repeated as often as required.

A configurable parameter of the sequence is $f_{1}$, which defines the frequency of the pattern. As a heterodyne approach is used later for phase unwrapping, the second frequency in the sequence is defined by $f_{2}=f_{1}-1$. Using this sequence design, phase recovery is possible at any position $k$ in the pattern stream $g_{k}$ captured by the camera by evaluating three neighboring patterns.

To summarize, at any given index $k$, the surface reflectivity component is estimated from the camera image sequence $g_{k}$ by

$$
a(x, y)=g_{k-1}(x, y)+g_{k+1}(x, y) .
$$

In a second step, the cosine modulated pattern component is recovered for both frequencies by calculating

$$
s_{1}(x, y)=\frac{\frac{1}{2}\left(g_{k-1}(x, y)-g_{k+1}(x, y)\right)}{a(x, y)} e^{i \phi_{k-1}}
$$

and

$$
s_{2}(x, y)=\frac{g_{k}(x, y)-a(x, y)}{a(x, y)} e^{i \phi_{k}},
$$

where $\phi_{k}$ describes the phase shift corresponding to each patterns. As illustrated in Fig. $5, \phi_{k}$ is either 0 or $\pi$, depending on its location in the pattern stream. Subsequently, two relative phase maps can be obtained from $s_{1}$ and $s_{2}$ using the Fourier method, followed by the phase unwrapping algorithm to obtain the absolute phase. The resulting $3-\mathrm{D}$ acquisition rate is correspondingly equal to the frame rate of the camera and projector. The proposed technique can also be used when projecting binary fringe patterns, as long as the bandpass filtering step is configured to suppress the higher fringe orders present in binary patterns.

\subsection{Data registration and merging}

A benefit of the high measurement rate of the sensor is that motion artifacts

in measurements are reduced compared to sensors with a lower acquisition rate. 


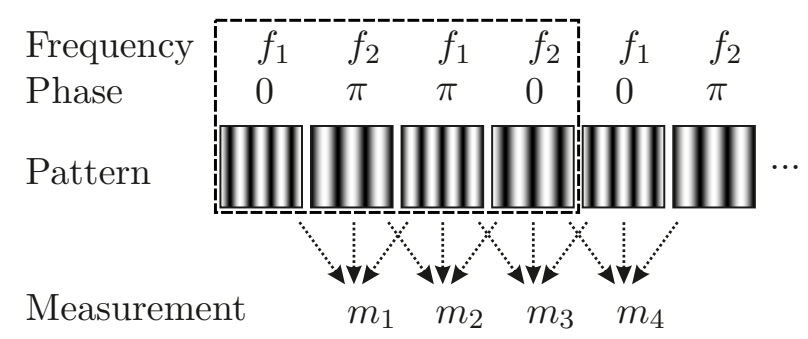

Figure 5. Interleaved projection sequence; Periodic projection of four patterns (dashed box) allows for the reconstruction of full-field 3-D measurements from any three consecutive camera images in the sequence.

Furthermore, it ensures a high number of overlapping 3-D surface points in consecutive datasets. This enables the use of odometry algorithms, which enable the automatic estimation of sensor pose for dataset alignment [32]. The algorithm employed performs the alignment based on geometrical features of the surface and does not require a-priori information about the environment. Sensor data can be provided in the form of either depth maps or 3-D point cloud data to be used with existing algorithms for this purpose.

In addition to the automatic registration of consecutively acquired 3-D point clouds into a common coordinate system, datasets are merged to reduce the influence of measurement noise. For this purpose, a modified implementation of the iterative closestpoint (ICP) algorithm [33] by Kjer and Wilm is used [34]. In summary, the algorithm iteratively minimizes the distance between closest-point matches in two datasets by estimating a rigid body transform. As a result, multiple datasets can be aligned to each other to cover a larger surface area than a single measurement, provided enough overlap is present in consecutive measurements. Additionally, sufficient geometrical features need to be present on the surface in order for the alignment to converge. In addition to the alignment of surface data, the estimated rigid body transforms describe the relative movement and rotation of the sensor head between measurements. As a result, the pose estimation allows to estimate the relative motion of the sensor head. When merging a large number of datasets, alignment errors accumulate over time, leading to a drift in the pose estimation result.

In order to reduce noise in measurements and reduce the amount of data to be stored, 3-D points in multiple datasets, which fall below a certain distance from each other, are merged to an averaged position. The number of measured points contributing to each data point after this merging procedure is stored. This information is further used for weighting during the registration procedure to increase robustness. Data points, which are present in multiple previously captured measurements, are thus preferably used to align newly captured 3-D datasets. Furthermore, by removing data points which are only present in a low number of measurements, outliers can be very effectively removed. 


\section{Experimental results}

In order to evaluate the performance of the sensor, measurements of reference objects are carried out at different locations in the measuring volume. In the first evaluation, a spherical reference object with a nominal diameter of $1 \mathrm{~mm}$ is positioned using a coordinate measuring machine. The second evaluation is aiming to assess the performance of the two introduced pattern sequences when applied for the measurements of objects in motion. The first reference object is a tactile ball probe with a nominal radius of $1 \mathrm{~mm}$. The surface of the spherical geometry has been treated by nitriding, a hardening process found in industrial applications. The radius has been calibrated using a coordinate measuring machine (CMM) of type ZMC550 by Zeiss (Oberkochen, Germany). Furthermore, a micro-contour standard by Alicona Imaging GmbH (Graz, Austria) is used in the evaluations. The contour of the standard exhibits multiple features, such as radii, steps and angles of different extent. Both reference objects feature a technical metal surface with direct reflection components. The results therefore allow for a realistic evaluation of measurement deviations in industrial applications, in contrast to best-case evaluations using diffusely coated reference objects often given for commercial structured light sensors.

The parameters used in the evaluations are derived from the guideline VDI/VDE

2634 Part 2, which defines different metrics for the assessment of optical areal scanners [35]. The three following metrics are used for the experiments:

- form measurement error

- size measurement error

- distance measurement error

The form measurement error is evaluated by calculating the standard deviation of the perpendicular distance of object points to the fitted reference geometry. In the context of the experiments performed, these are a sphere and a cylinder. Additionally, the probe error is obtained by comparing the measured value of the fitted reference object to the calibrated value. For the spherical and cylindrical object, these are the calibrated radii. Furthermore, the spacing error is used in the evaluation of the conventional measuring mode and defined by the deviation of the distance of fitted sphere centers to the reference values.

\subsection{Conventional measuring mode}

For the evaluation of measurement deviations in the conventional measuring mode, the previously described spherical reference object measured at different locations in the measuring volume. A coordinate measuring machine of type Werth Videocheck IP (Giessen, Germany) is used to accurately position the sphere and obtain reference measurements of the sphere centre locations. In total, 27 centre positions are measured in a volume with a depth of $2 \mathrm{~mm}$ and $4.5 \mathrm{~mm}$ width and height. A sensor head with a triangulation base of length $6 \mathrm{~mm}$ and a working distance of $10 \mathrm{~mm}$ is attached to the 


\begin{tabular}{|l|c|}
\hline Max. pattern frequency & 50 \\
\hline Pattern count & 8 \\
\hline Exposure durations & $10.2 \mathrm{~ms}, 40.8 \mathrm{~ms}, 163.2 \mathrm{~ms}$ \\
\hline Total acquisition time & $1.9 \mathrm{~s}$ \\
\hline
\end{tabular}

Table 2. Evaluation of feature parameters for hand-guided measurement.
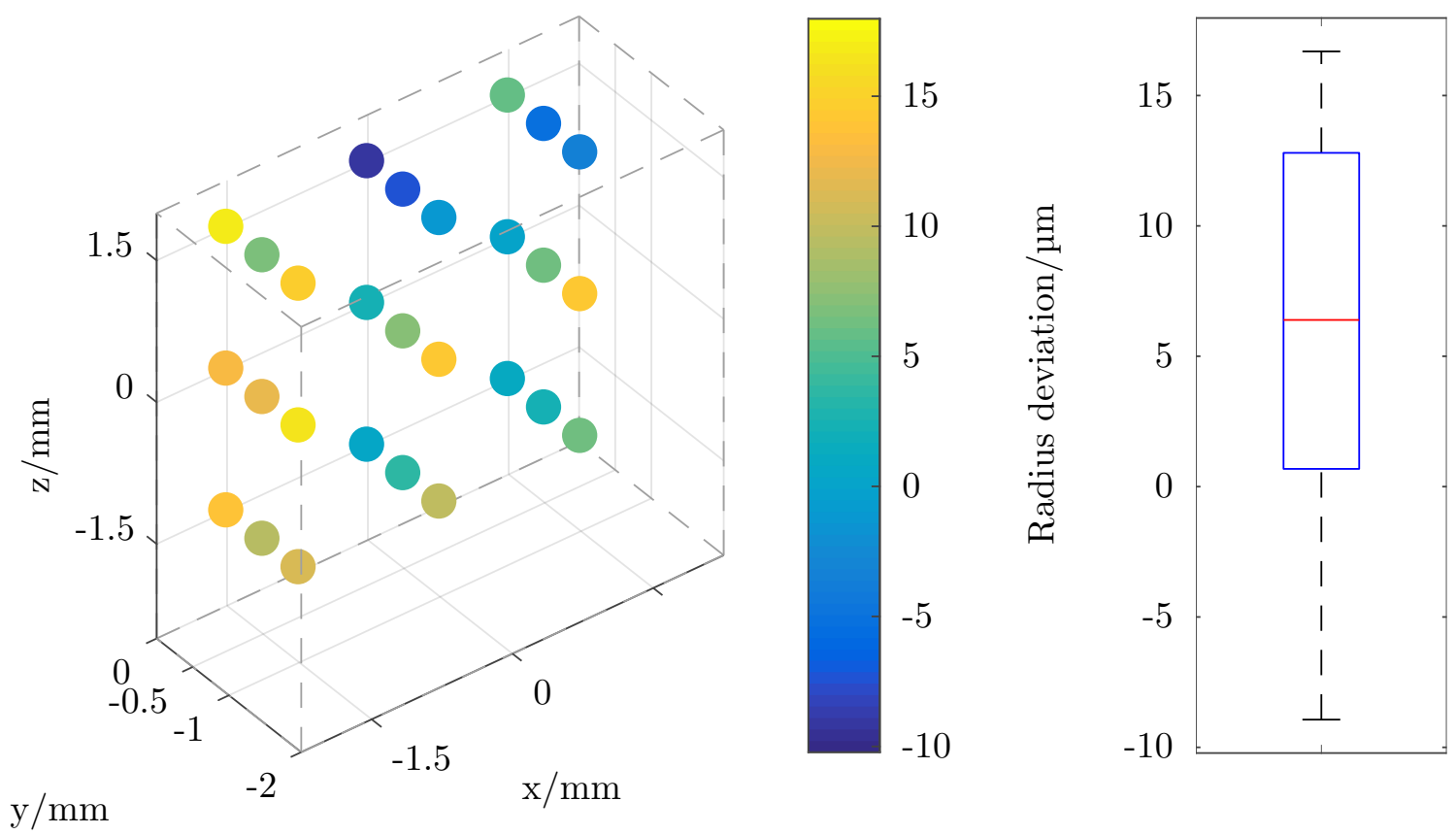

Figure 6. Size measurement errors at different positions.

measuring endoscope, i.e. the design shown in Fig. 3. The configured measurement parameters are shown in Tab. 2.

Fig. 6 shows the size measurement errors for the spheres measured at different positions in the measuring volume. Deviations to the calibrated radius range from approximately $-10 \mu \mathrm{m}$ to $16 \mu \mathrm{m}$. An evaluation of the form measurement errors is shown in Fig. 7. In the centre of the volume, the standard deviation of form deviations are below $2.5 \mathrm{\mu m}$, while the deviations increase to the edges of the evaluated volume. Standard deviations are below $4.0 \mu \mathrm{m}$ at all measurement positions. Distance measurement errors between all measured sphere centre positions are plotted in Fig. 8. Relative errors are slightly higher for the shortest distances measured and fall below $2 \%$ for larger distances.

\subsection{Continuous measuring mode}

For the evaluation of the continuous measuring mode, the convex cylindrical feature with a nominal radius of $1 \mathrm{~mm}$ on the contour standard is chosen as a reference object. 

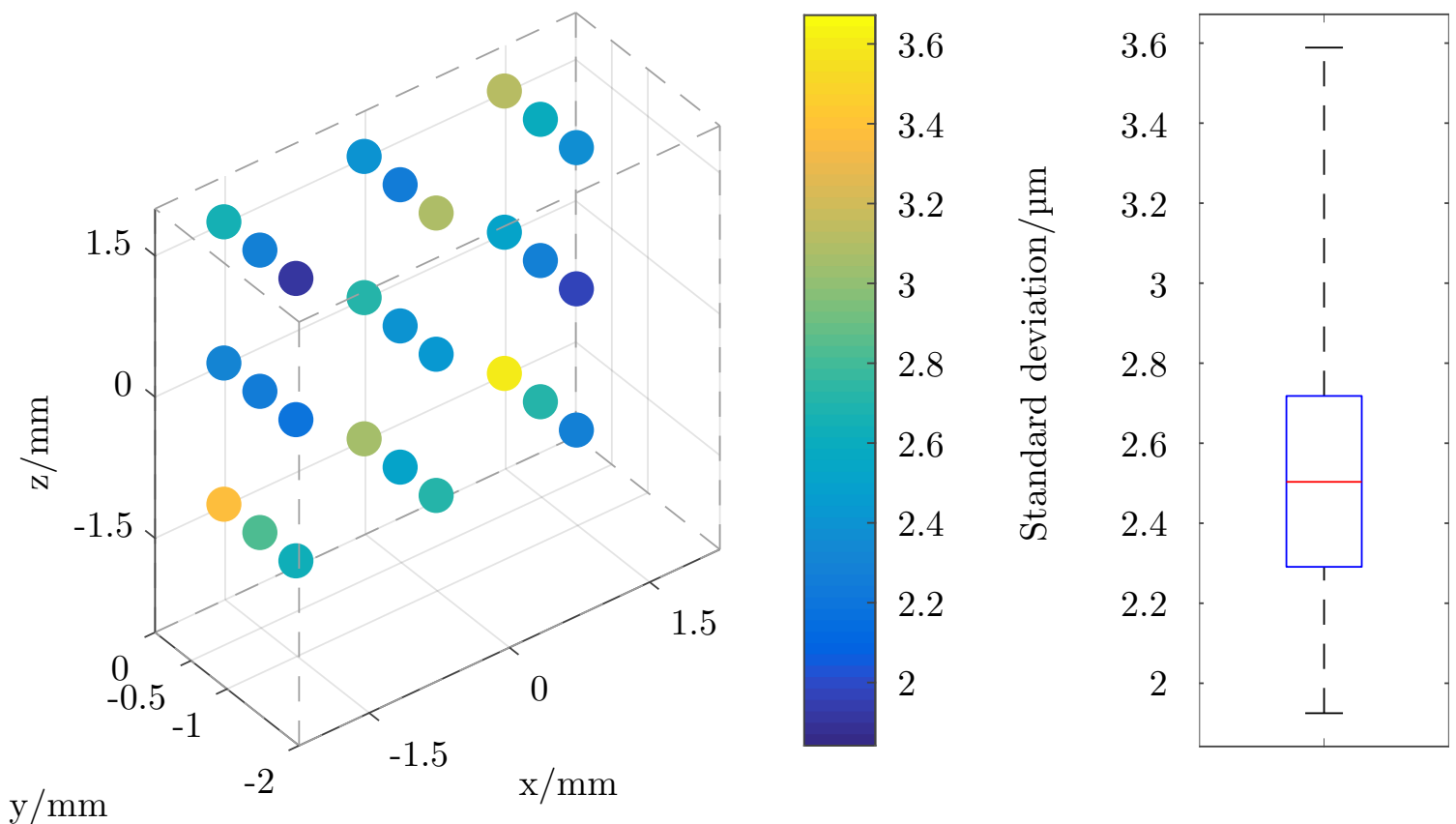

$\mathrm{y} / \mathrm{mm}$

Figure 7. Form measurement errors at different positions.

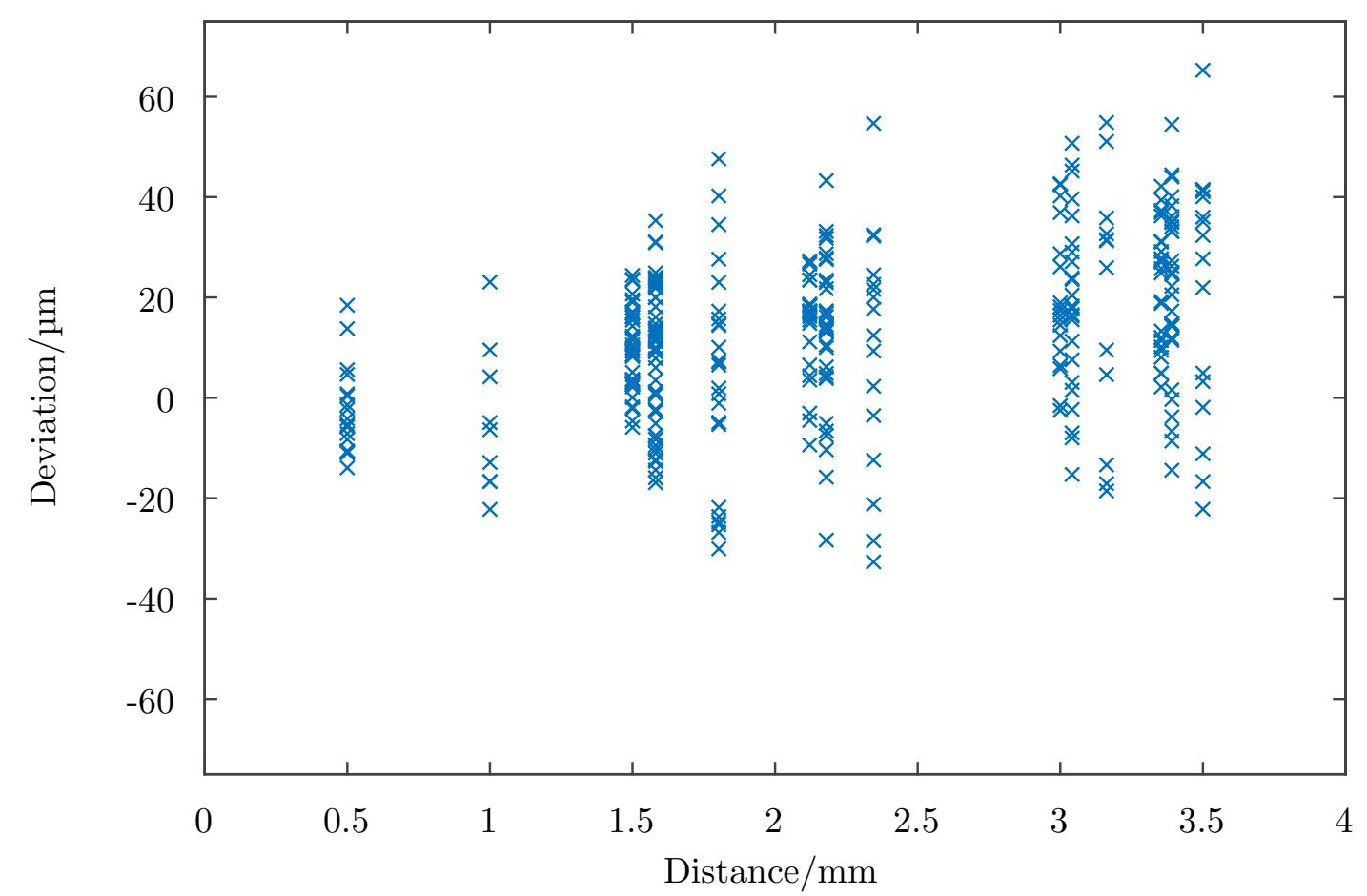

Figure 8. Distance measurement errors between all measured sphere positions. 


\begin{tabular}{|l|c|c|}
\hline Sequence type & conventional & continuous \\
\hline Max. frequency & 50 & 25 \\
\hline Proj. patterns & 10 & 10 \\
\hline Acquisition time & $75.8 \mathrm{~ms}$ & $75.8 \mathrm{~ms}$ \\
\hline Point clouds & 1 & 8 \\
\hline
\end{tabular}

Table 3. Evaluation of feature parameters for handheld measurement.

In order to induce motion with reproducible velocities, the micro-contour standard is mounted onto a linear stage. Measurements are performed at three different velocities using both previously described measuring modes.

A sensor head with a triangulation base of length $6 \mathrm{~mm}$ and a working distance of $20 \mathrm{~mm}$ is attached to the measuring endoscope, as shown in Fig. 3. Compared to the evaluations presented in Section 4.1, this results in half the triangulation angle, while the depth of the measuring volume is approximately tripled and the field diameter doubled.

Measurement parameters have been defined to the values shown in Tab. 3 for the different modes. As the continuous mode is using fewer additional patterns for unwrapping compared to the conventional mode, the highest frequency needs to be reduced. This results in a slight reduction in robustness towards noise as well as lateral resolution. In order to achieve comparable results, the total acquisition time and number of total patterns has been set identically for both modes. Camera and projector operate at a rate of $132 \mathrm{~Hz}$ in this configuration. As described in Section 3.3, individual measurements obtained by the continuous mode in one run are automatically registered and merged. The complete measurement and analysis procedure is repeated 25 times for both modes.

Analysis of the size probing error at three different velocities for both modes is shown in Fig. 9. For the non-moving cylinder, the measurement deviations are slightly lower when using the conventional measuring mode, while both modes obtain results with a maximum deviation of less than $10 \mu \mathrm{m}$. At a velocity of $0.5 \frac{\mathrm{mm}}{\mathrm{s}}$, size probing deviations significantly increase in the conventional measuring mode, ranging from approximately $-10 \mu \mathrm{m}$ to $20 \mu \mathrm{m}$. When using the continuous measuring mode at this velocity, form deviations increase to approximately $10 \mu \mathrm{m}$. In comparison to the conventional mode, the range of deviations is smaller. At a velocity of $2.0 \frac{\mathrm{mm}}{\mathrm{s}}$, which corresponds approximately to a displacement of $20 \%$ of the field diameter per second, no valid measurements are obtained in the conventional mode. Using the continuous mode, the range of deviations increases to approximately $-30 \mu \mathrm{m}$ to $20 \mu \mathrm{m}$.

The standard deviation of form deviations to the fitted cylinder are shown in Fig. 10. For the non-moving reference object, form deviations are lower in the conventional measuring mode, indicating less noise in the measurement data. At higher velocities, the standard deviation increases when measuring in the conventional mode, due to artefacts emerging from the object's motion. Using the continuous measuring mode, the standard deviation of form deviations increases only slightly with increasing velocity. 


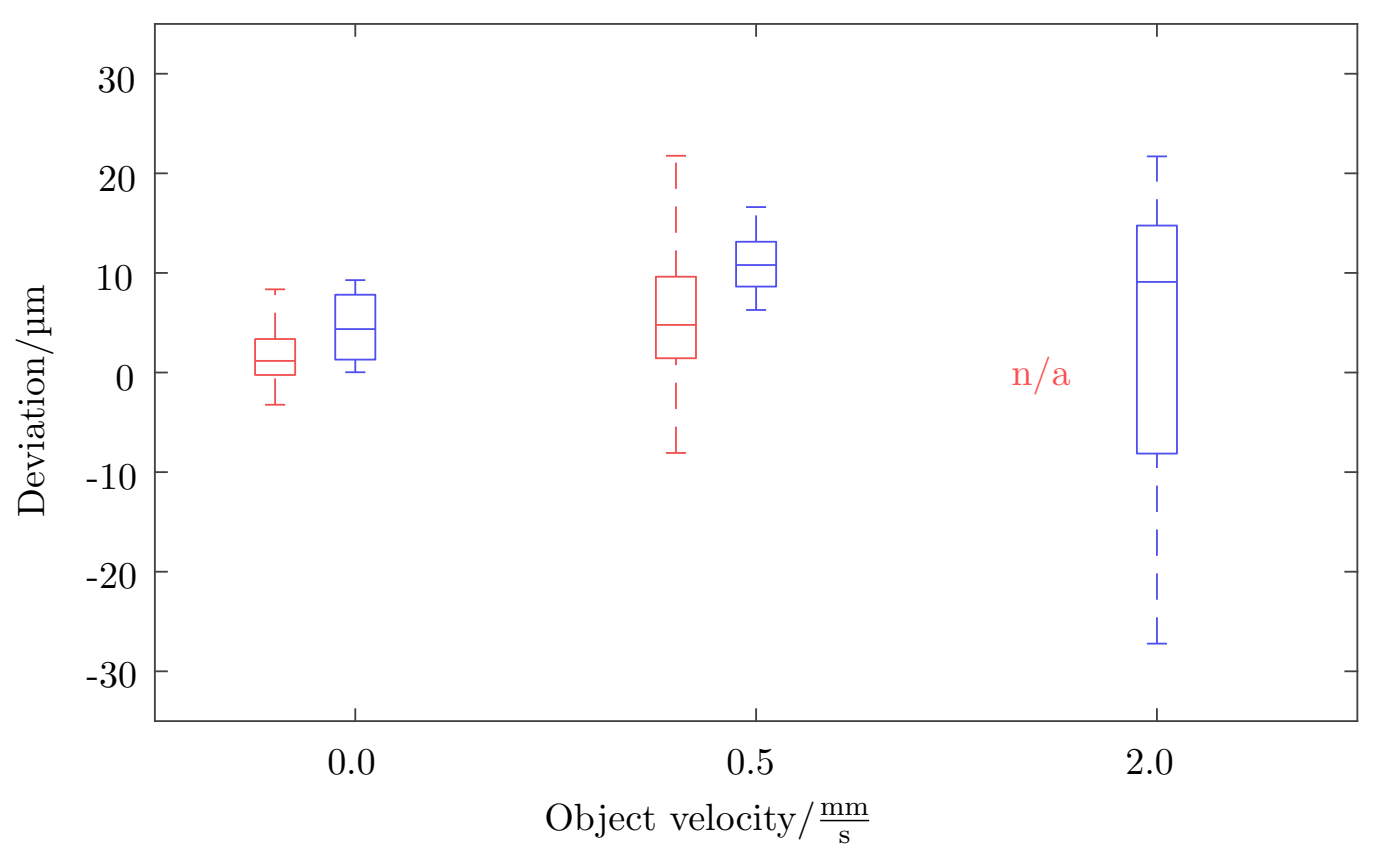

Figure 9. Size measurement errors for moving cylinder. Left: Conventional mode; Right: Continuous mode.

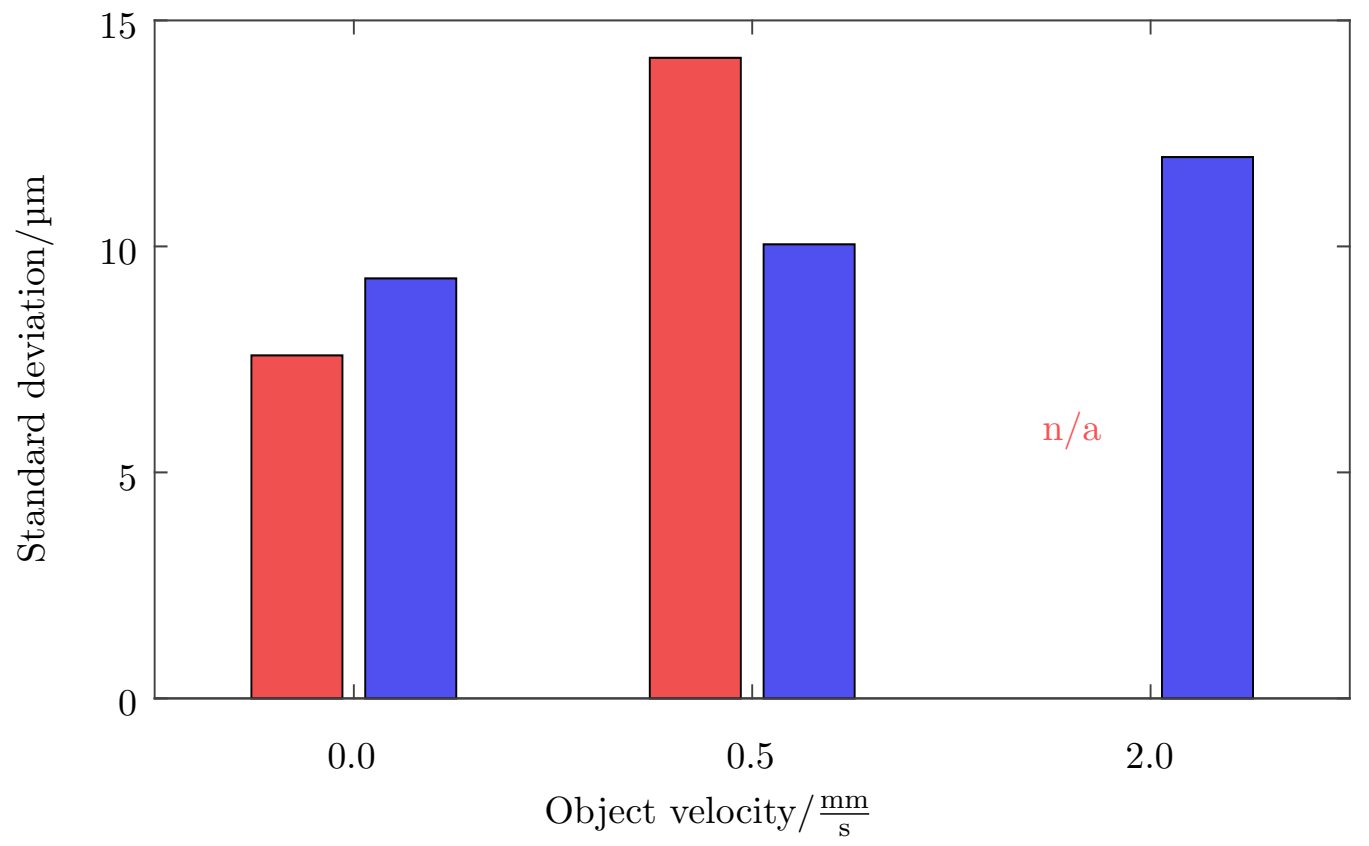

Figure 10. Form measurement errors for moving cylinder. Left: Conventional mode; Right: Continuous mode. 


\begin{tabular}{|l|c|}
\hline Max. frequency & 15 \\
\hline Projection rate & $172 \mathrm{~Hz}$ \\
\hline Merging distance & $75 \mu \mathrm{m}$ \\
\hline Acquisition duration & $26 \mathrm{~s}$ \\
\hline
\end{tabular}

Table 4. Measurement parameters for hand-guided measurement.

\begin{tabular}{|l|c|c|c|}
\hline Feature & Reference & Measured & Form deviation \\
\hline Cylinder & $498.7 \mu \mathrm{m}$ & $476.3 \mu \mathrm{m}$ & $10.9 \mu \mathrm{m}$ \\
\hline Cylinder & $998.7 \mu \mathrm{m}$ & $1027.0 \mu \mathrm{m}$ & $12.8 \mu \mathrm{m}$ \\
\hline Angle & $89.96^{\circ}$ & $87.4^{\circ}$ & $7.2 \mu \mathrm{m}$ \\
\hline Step height & $1000.5 \mu \mathrm{m}$ & $995.3 \mu \mathrm{m}$ & $7.2 \mu \mathrm{m}$ \\
\hline Step height & $500.4 \mu \mathrm{m}$ & $480.0 \mu \mathrm{m}$ & $8.0 \mu \mathrm{m}$ \\
\hline
\end{tabular}

Table 5. Evaluation of feature parameters for hand-guided measurement.

In a second experiment, the sensor is moved by hand along the contour of the geometry standard. The goal of the experiment is to evaluate the performance of the continuous measuring mode for this application as well as to assess the robustness against more irregular motion. Similar to the previous evaluation, the sensor head with a triangulation base length of $6 \mathrm{~mm}$ and lenses with a working distance of $20 \mathrm{~mm}$ are used. The parameters of the measurements are shown in Tab. 4. Merging distance defines the parameter for the algorithm outlined in Section 3.3 and limits the lateral resolution of point clouds.

The measurement result is shown in Fig. 11. The measured geometry is shown in the form of a point cloud in the upper part of the figure, while the reconstructed trajectory of the sensor head is drawn as a line in the lower part of the image. Tab. 5 shows evaluations for features on the contour standard. Form deviations are given as the standard deviation to the fitted reference geometry, i.e. cylinder or plane. Compared to the previous evaluations, deviations are increased. However, despite the irregular motion and further reduction in lateral resolution, the parameters of the reference geometries can be estimated with less than $10 \%$ deviation.

\section{Discussion}

The compact designs of the sensor head allow to adapt to different applications, for example by varying size and working distance of the endoscope tip. By using only passive components, the sensor is more robust towards increased temperatures compared to chip-on-tip cameras. Moreover, the endoscope can be positioned in electromagnetic fields and fluids. Further reduction of the size of sensor heads is possible by permanently connecting lenses and fibres by adhesives.

The measurement results demonstrate the adaptability of the sensor to different measurement tasks. A comparison using an earlier version of the sensor already 


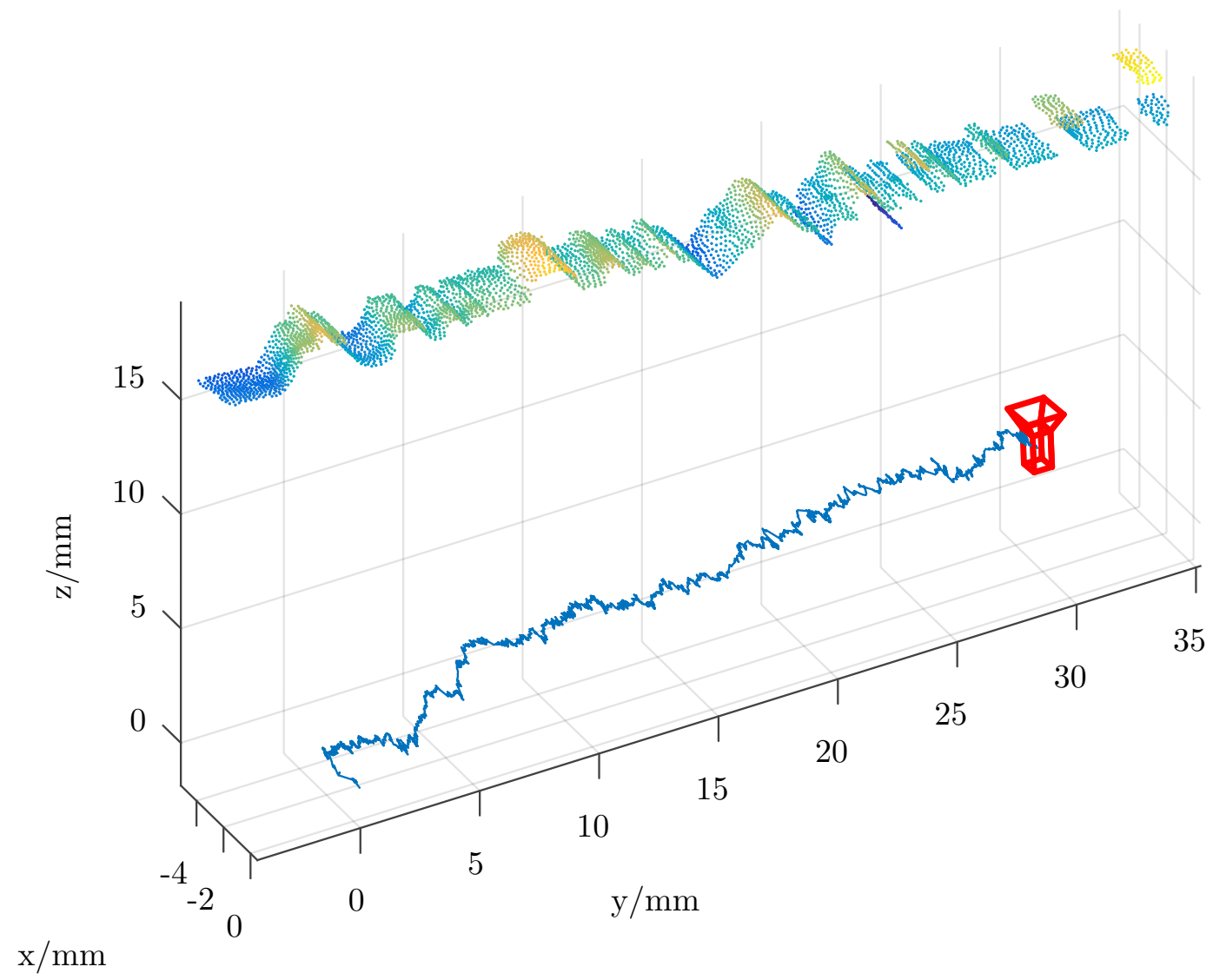

Figure 11. Registered and merged point cloud data and reconstructed sensor head trajectory

demonstrated results comparable to non-endoscopic structured light sensors for micro geometries (GFM MicroCad) [36]. The evaluations performed in Section 4.1 using the conventional measuring mode and a sensor head with a working distance of $10 \mathrm{~mm}$ demonstrate the capability of the sensor to achieve accurate results at arbitrary positions in the measurement volume. Maximum deviations to the calibrated radius of a sphere amount to $15 \mu \mathrm{m}$. Despite specular highlights on the reference objects, the standard deviation of form deviation errors does not exceed $4 \mu \mathrm{m}$ over a depth range of $2 \mathrm{~mm}$.

The evaluations in Section 4.2 are performed at shorter acquisition durations of around $75 \mathrm{~ms}$. Furthermore, the triangulation angle is reduced compared to the results in Section 4.1 due to the use of lenses with a larger working distance of $20 \mathrm{~mm}$. Despite these constraints, deviations to the calibrated radius of a reference cylinder do not exceed $10 \mu \mathrm{m}$ in the centre of the measuring volume for a non-moving object. When motion between sensor and measuring object cannot be avoided, the continuous measuring mode is capable of providing reliable results, with the extent of measurement deviations depending on the velocity. The proposed projection sequence allows for the acquisition of full-field 3-D measurements at a rate identical to the frame rate of the employed camera 
without constraints on the specimen's geometry. In the continuous measuring mode, cylindrical geometries can be measured at velocities corresponding to approximately $20 \%$ of the measurement field diameter per second. Compared to the conventional measuring mode, the reduced number of patterns results in a loss of lateral resolution and slight increase in noise in the measurement data. However, even when moving the sensor by hand, small elements such as the $0.1 \mathrm{~mm}$ radius are still clearly discernible in the measurement data. Moreover, measurements exhibit low noise owing to the registration and merging of a high number of individual measurements. Owing to the very short acquisition time and the larger measuring volume, measurement noise is higher than in the conventional measuring mode, with a standard deviation of form deviations below $13 \mu \mathrm{m}$.

The added ability of estimating the relative pose of the sensor head allows for an assisted navigation in enclosed spaces. However, two limitations of the tracking procedure need to be noticed. Currently, the registration algorithm relies on measured geometrical features to align consecutively acquired 3-D data and is therefore not requiring texture features. If no distinctive features are present, alignment and pose estimation may fail. Furthermore, pose estimation errors accumulate when moving the sensor head over larger distances, resulting in drift errors. In the future robustness could be further improved by additionally tracking intensity features, which have been previously used to improve the registration process [37]. Owing to the small dimensions of the sensor head and motion, comparison to ground-truth pose data cannot be provided. However, pose estimation errors are correlated to geometry alignment errors for odometry algorithms and thus affect the measured geometry. The comparison of measured radii and angles to their reference values indicate an alignment without larger deviations.

As described in Section 1, maintenance processes for example of air-plane engines require the hand-guided insertion of endoscopes. Similar to commercially available solutions, this currently requires the endoscope to be in contact with the inspected cavity.

In summary, the results demonstrate the capability of the new sensor to obtain accurate measurements even under the condition of irregular motion of the tip. Owing to the flexible design of the sensor head, measuring modes can be changed in less than $100 \mathrm{~ms}$, allowing for adjustment depending on the currently encountered measurement task.

\section{Summary and outlook}

A new endoscopic 3-D sensor was presented, which employs two fibre-optical endoscopes to enable the measurement of geometries in enclosed spaces. Using conventional phaseshift sequences, the sensor is capable of a depth acquisition rate of up to $20 \mathrm{~Hz}$. When using a newly introduced periodic phase-shift sequence, the acquisition rate can be increased to up to $180 \mathrm{~Hz}$ in the current sensor configuration. This enables the 
measurement of objects even in the presence of relative motion to the sensor head, as demonstrated by detailed evaluation. Furthermore, a hand-guided measurement of micro-geometries with odometry for the estimation of sensor head location and orientation was presented.

Detailed evaluations using a spherical reference object and a CMM for accurate positioning show form deviations with a standard deviation of less than $5 \mu \mathrm{m}$ and radius deviations of less than $15 \mu \mathrm{m}$ throughout the measurement volume at a working distance of $10 \mathrm{~mm}$. In summary, the sensor exceeds existing measuring endoscopes in both accuracy and measuring speed.

The sensor is applicable to a multitude of industrial applications. Examples are the in-situ inspection of tools in production processes or the inspection of engines in maintenance processes. Further potential applications can be found in the medical field, for example as a dental scanner.

In the future, the high acquisition rate of simultaneous depth and intensity images could be used in combination with a SLAM algorithm in order to provide improved navigation in enclosed areas. For example, the inspection of jet engines could benefit from the ability to register measurements to CAD reference data based on sensor head tracking data. In order to qualify the sensor for this application, ground truth data for the sensor position could be obtained for example by using electromagnetic tracking.

Further research will focus on further miniaturization of sensor heads. This will enable the integration in a continuum robot, which would allow the endoscope to navigate around obstacles. Miniaturization of these types of robots is currently the topic of on-going research [38]. The described approaches for simultaneous localization and measurement could be used to achieve closed loop control of the robot end-effector position.

\section{Acknowledgement}

The authors would like to thank the German Research Foundation (DFG) for funding the project B6 "Endoscopic geometry inspection" within the Collaborative Research Center (CRC) / TR 73 .

\section{References}

[1] Raj B, Jayakumar T and Thavasimuthu M 2002 Practical non-destructive testing (Woodhead Publishing)

[2] Schwenke H, Neuschaefer-Rube U, Pfeifer T and Kunzmann H 2002 CIRP Annals - Manufacturing Technology 51 685-699 ISSN 00078506

[3] Frankowski G and Hainich R 2009 DLP-based 3D metrology by structured light or projected fringe technology for life sciences and industrial metrology Emerging digital micromirror device based systems and applications vol 7210 (International Society for Optics and Photonics) p 72100C

[4] Weckenmann A, Krämer P and Hoffmann J 2007 Manufacturing metrology-state of the art and prospects Proc. ISMQC pp 1-8

[5] Sansoni G, Trebeschi M and Docchio F 2009 Sensors 9 568-601 
[6] Drury C G and Watson J Good practices in visual inspection

[7] Weckenmann A, Krämer P and Hoffmann J 2007 Manufacturing metrology-state of the art and prospects Proc. ISMQC pp 1-8

[8] Geng J and Xie J 2014 IEEE Sensors Journal 14 945-960 ISSN 1530-437X

[9] Noonan D P, Mountney P, Elson D S, Darzi A and Yang G Z 2009 A stereoscopic fibroscope for camera motion and 3D depth recovery during minimally invasive surgery IEEE International Conference on Robotics and Automation (IEEE) pp 4463-4468 ISBN 978-1-4244-2788-8

[10] Penne J, Höller K, Stürmer M, Schrauder T, Schneider A, Engelbrecht R, Feußner H, Schmauss B and Hornegger J 2009 Time-of-flight 3-D endoscopy Medical Image Computing and ComputerAssisted Intervention-MICCAI 2009 (Springer) pp 467-474

[11] Hasegawa K, Noda K and Sato Y 2002 Electronic endoscope system for shape measurement Proceedings / 16th International Conference on Pattern Recognition ed Kasturi R (IEEE Computer Society) pp 761-764 ISBN 0-7695-1695-X

[12] Pulwer S, Steglich P, Villringer C, Bauer J, Burger M, Franz M, Grieshober K, Wirth F, Blondeau J, Rautenberg J, Mouti S and Schrader S 2016 Triangulation-based 3D surveying borescope Proc. SPIE (SPIE Proceedings vol 9890) ed Gorecki C, Asundi A K and Osten W (SPIE) p 989009

[13] Industrial Group 26/06/2015 New KARL STORZ MULTIPOINT measuring videoscope features impressive minimal diameter and a 'see \& measure' function

[14] GE Inspection Technologies Videoprobe 3D measurement handbook: A guide to advanced 3D measurement technologies, techniques and applications for video borescopes used in remote visual inspection applications.

[15] Korczewski Z 2013 Polish Maritime Research 20 ISSN 1233-2585

[16] Chen X, Reichenbach K L and Xu C 2008 Optics express 16 21598-21607

[17] Matthias S, Kästner M and Reithmeier E 2016 Advanced Optical Technologies 5 439-449 ISSN $2192-8584$

[18] Matthias S, Kästner M and Reithmeier E 2017 Fiber-optic fringe projection with crosstalk reduction by adaptive pattern masking Proc. SPIE vol 10117 pp 101170A-1

[19] Hartley R I and Zisserman A 2004 Multiple View Geometry in Computer Vision 2nd ed (Cambridge University Press, ISBN: 0521540518)

[20] Brown D C 1966 Photometric Engineering 32 444-462

[21] Salvi J, Fernandez S, Pribanic T and Llado X 2010 Pattern Recognition 43 2666-2680

[22] Takeda M and Mutoh K 1983 Applied Optics 223977 ISSN 0003-6935

[23] Zhang Z 2012 IEEE Multimedia 19 4-10 ISSN 1070-986X

[24] Zuo C, Huang L, Zhang M, Chen Q and Asundi A 2016 Optics and lasers in engineering 85 84-103

[25] Sansoni G, Carocci M and Rodella R 1999 Applied optics 38 6565-6573

[26] Larkin K G and Oreb B F 1992 JOSA A 9 1740-1748

[27] Peng T, Gupta S K and Lau K 2005 Computer-Aided Design and Applications 2 737-746

[28] Mann S and Picard R W 1995 On being 'undigital' with digital cameras: Extending dynamic range by combining differently exposed pictures Proceedings of ISET pp 442-448

[29] Matthias S, Kästner M and Reithmeier E 2017 Pattern sequences for fast absolute phase retrieval with application in the handheld operation of structured light sensors Proceedings of 59th IWK

[30] Creath K 1988 Progress in optics 26 349-393

[31] Towers C E, Towers D P and Jones J D 2003 Optics letters 28 887-889

[32] Nuchter A, Surmann H, Lingemann K, Hertzberg J and Thrun S 2004 6D SLAM with an application in autonomous mine mapping Robotics and Automation, 2004. Proceedings. ICRA'04. 2004 IEEE International Conference on vol 2 (IEEE) pp 1998-2003

[33] Rusinkiewicz S and Levoy M 2001 Efficient variants of the ICP algorithm Proceedings / Third International Conference on 3-D Digital Imaging and Modeling (LosAlamitos, Calif.: IEEE Computer Soc) pp 145-152 ISBN 0-7695-0984-3

[34] Kjer H M and Wilm J 2010 Evaluation of surface registration algorithms for PET motion correction 
B.s. thesis Technical University of Denmark, DTU, DK-2800 Kgs. Lyngby, Denmark

[35] VDI/VDE-Gesellschaft Mess- und Automatisierungstechnik VDI/VDE 2634 part 2 - optical 3-D measuring systems - optical systems based on areal scanning

[36] Matthias S, Loderer A, Koch S, Gröne M, Kästner M, Hübner S, Krimm R, Reithmeier E, Hausotte T and Behrens B A 2016 Production Engineering 10 51-61 ISSN 0944-6524

[37] Li S and Lee D 2016 IEEE Robotics and Automation Letters 1 992-999

[38] Amanov E, Granna J and Burgner-Kahrs J 2017 Toward improving path following motion: Hybrid continuum robot design Robotics and Automation (ICRA), 2017 IEEE International Conference on pp 4666-4672 